\title{
Kinematic Synthesis of Stephenson III Six-bar Function Generators
}

\author{
Mark M. Plecnik, J. Michael McCarthy \\ Robotics and Automation Laboratory \\ Department of Mechanical and Aerospace Engineering \\ University of California, Irvine \\ Irvine, California 92697
}

\begin{abstract}
This paper presents a direct solution of the kinematic synthesis equations for Stephenson III six-bar function generators to achieve as many as 11 accuracy points. The approach is similar to that used to design Stephenson II function generators, except additional algebraic manipulations reduce the system to a multihomogeneous degree of 55,050,240. A numerically general multihomogeneous homotopy was used to obtain 834,441 nonsingular solutions, which were then used to construct an efficient parameter homotopy for specific tasks consisting of 11 accuracy points. The thousands of linkage solutions found by this parameter homotopy are sorted and analyzed to verify nonbranching movement through the specified task positions. An example is presented of a function generator that creates a specified torque-angle profile for a dynamic wrist splint that cancels the effects of spasticity in the wrists of stroke survivors.
\end{abstract}

Keywords:

linkage synthesis, function generator, six-bar

\section{Introduction}

This paper presents a direct solution of the kinematic synthesis equations for Stephenson III six-bar linkages that yield function generators that

Email addresses: mplecnik@uci.edu (Mark M. Plecnik), jmmccart@uci.edu (J. Michael McCarthy)

Preprint submitted to Mechanism and Machine Theory

September 29, 2015

(C) 2015. This manuscript version is made available under the Elsevier user license http://www.elsevier.com/open-access/userlicense/1.0/ 
can achieve as many as 11 accuracy points. It is shown that the synthesis equations reduce to a polynomial system with a multihomogeneous degree $55,050,240$, unlike the synthesis equations for Stephenson II function generators, Plecnik and McCarthy (2015). The polynomial homotopy software BERTini, Bates et al. (2013), was used to solve a numerically general version of the synthesis equations running on $512 \times 2.6 \mathrm{GHz}$ cores of the Gordon system at the San Diego Supercomputer Center. The result was 834,441 nonsingular solutions which were used to construct parameter homotopies for specific function generator synthesis tasks. The parameter homotopies obtained candidate designs in under an hour running on $64 \times 2.2 \mathrm{GHz}$ machines of the UC Irvine High Performance Computing Cluster. These results are the first to obtain designs for six-bar function generators for as many as 11 accuracy points by direct solution of the synthesis equations.

The synthesis equations yield thousands of candidate linkage designs which are analyzed to find those that achieve the required task in a single configuration and without passing through a singularity. This requires identification of the function generator cognates, Dijksman (1976), among the design candidates, and a direct numerical analysis of each candidate design. It can happen that a cognate solution does not appear in the synthesis results due to numerical issues, in which case those cognates are constructed and added to the synthesis results. The result is a set of linkages that achieve the specified function generation task and are free of branch and circuit defects.

In order to illustrate the results, an 11 position task is presented that arises from the design of a torque-balancing assistive wrist splint which is intended to cancel the stiffness in the wrists of stroke survivors who suffer from spasticity. The detailed design of this device is the focus of current research, Bissell (2014), where integral to the design is a six-bar function generator that can transform a linear spring into a specified torque-angle profile. The input-output profile required to create the necessary torque is a nonlinear function of angular displacement that is effectively approximated by 11 accuracy points.

\section{Literature Review}

A six-bar linkage consists of four binary links, that is links with two joints, and two ternary links, links with three joints. These systems form two distinct topologies known as Watt and Stephenson six-bar linkages. The Stephenson six-bar linkages have the property that the two ternary links are 
separated by the binary links, while the Watt topology has the ternary links connected to each other, Tsai (2000).

The kinematic synthesis of six-bar function generators is an extension of the original work by Freudenstein (1954), where the loop equations of the system are formulated in each of the configurations specified by the required input-output angles. Also see Hartenberg and Denavit (1964). The result is a set of polynomial equations that are solved to determine the dimensions of the linkage.

McLarnan (1963) formulated this problem for both Stephenson II and Stephenson III function generators and found solutions for eight accuracy points using the Newton-Raphson method on an IBM 704 computer. In 1994, Dhingra et al. (1994) returned to this problem and solved the synthesis equations for both the Stephenson II and Stephenson III six-bar function generators for nine accuracy positions using a polynomial homotopy algorithm. Also see Akçali (1995); Liu et al. (1995); Simionescu and Alexandru (1995). This paper is the first to provide a direct solution of the synthesis equations for the 11 accuracy point problem, which is the maximum that can be achieved for a six-bar function generator.

Our approach is different from recent efforts that use optimization techniques to find solutions to the synthesis equations, Root and Ragsdell (1976); Blackett (2001). Optimization relies on a starting set of dimensions for the linkage, that is refined using the synthesis equations, Hwang and Chen (2010); Sancibrian (2011), to minimize the difference between the existing inputoutput function of the linkage and the desired function. Bulatović et al. (2013) introduced the Cuckoo Search algorithm, and Shiakolas et al. (2005) used a method known as differential evolution to find solutions. We find exact solutions to the synthesis equations using a polynomial homotopy solver, which means that we obtain literally thousands of solutions, because the degree of the polynomial system is so large.

\section{Synthesis Equations for the Stephenson III}

The Stephenson III six-bar linkage, Figure 1, has a ternary link as its ground link, and consists of two loops defined by joint coordinates: (i) $A D G C$, and (ii) $A D H F B$. The ground pivots $A$ and $B$ are used as the centers of $N$ coordinated angles $\phi_{j}$ and $\psi_{j}, j=0, \ldots, N-1$. The joint angles of $A$ and $C$ are part of a four-bar sub-loop, which means their coordinated movement is defined by four-bar loop equations. 


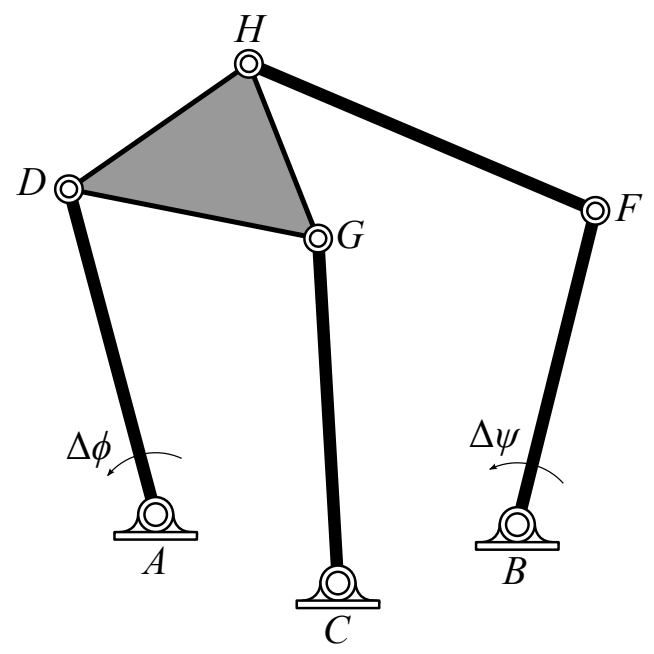

Figure 1: A Stephenson III six-bar function generator.

The planar kinematics of linkages is conveniently formulated using complex numbers, Erdman et al. (2001). The scale, orientation, and location of a reference configuration for the linkage is defined in the plane by selecting $A=0+0 i$ and $D=1+0 i$. The coordinates of the remaining joints $B, C$, $F, G$ and $H$ are calculated by solving the synthesis equations.

Introduce $\left(\Delta \phi_{j}, \Delta \psi_{j}\right), j=1, \ldots, N-1$ as the required input-output angles measured from the reference configuration, Figure 2(a), where

$$
\left(\Delta \phi_{j}, \Delta \psi_{j}\right)=\left(\phi_{j}-\phi_{0}, \psi_{j}-\psi_{0}\right), \quad j=1, \ldots, N-1 .
$$

The synthesis equations for the Stephenson III linkage are formed from the loop equations for each set of specified input-output angles.

The coordinates of the moving pivots of the linkage in each task configuration are related to their coordinates in the reference configuration by the equations,

$$
\begin{aligned}
D_{j} & =A+e^{\Delta \phi_{j}}(D-A), \\
F_{j} & =B+e^{\Delta \psi_{j}}(F-B), \\
G_{j} & =A+e^{\Delta \phi_{j}}(D-A)+e^{\Delta \mu_{j}}(G-D), \\
H_{j} & =A+e^{\Delta \phi_{j}}(D-A)+e^{\Delta \mu_{j}}(H-D), \quad j=1, \ldots, N-1 .
\end{aligned}
$$




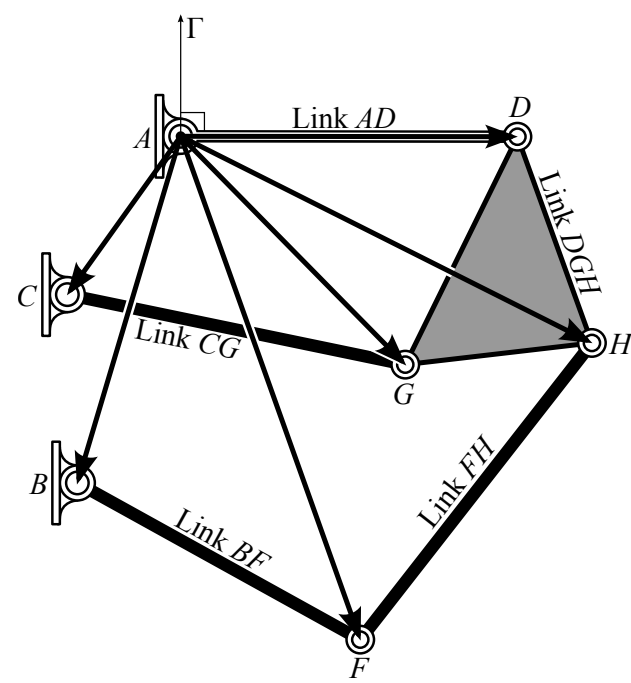

(a)

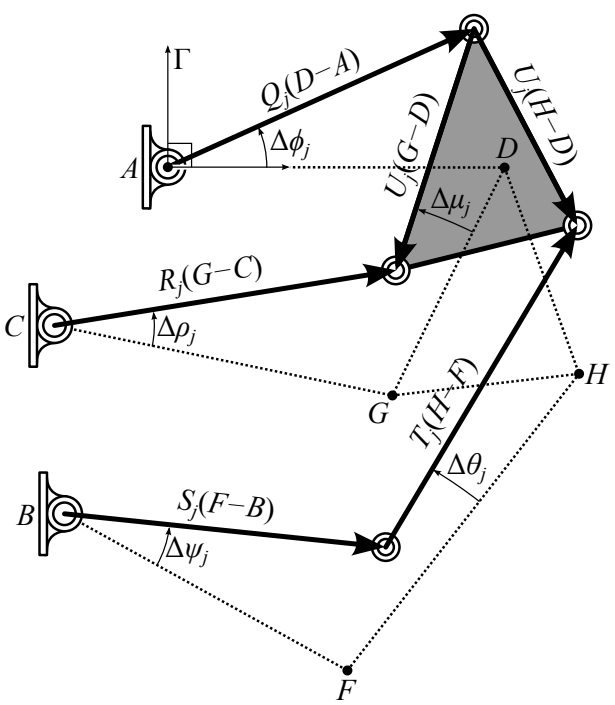

(b)

Figure 2: (a) The reference configuration for the synthesis of a Stephenson III function generator. (b) The specified angles of the Stephenson III function generator are measured relative to the reference configuration.

For convenience, introduce the notation,

$$
\begin{aligned}
& Q_{j}=e^{i \Delta \phi_{j}}, \quad R_{j}=e^{i \Delta \rho_{j}}, \quad S_{j}=e^{i \Delta \psi_{j}}, \\
& T_{j}=e^{\Delta \theta_{j}}, \quad U_{j}=e^{\Delta \mu_{j}}, \quad j=1, \ldots, N-1 \text {. }
\end{aligned}
$$

Notice that $Q_{j}$ and $S_{j}$ are defined by the required input-output angle pairs. The remaining joint angles are unknowns that satisfy the normalization conditions,

$$
R_{j} \bar{R}_{j}=1, \quad T_{j} \bar{T}_{j}=1, \quad U_{j} \bar{U}_{j}=1, \quad j=1, \ldots, N-1,
$$

where the overbar denotes the complex conjugate.

The loop equations for the Stephenson III six-bar linkage are obtained by evaluating $G_{j}-C$ and $H_{j}-F_{j}$ relative to the initial configuration. This 
yields two sets of complex conjugate loop equations, Wampler (1996),

$$
\begin{aligned}
& \mathcal{L}_{j}: R_{j}(G-C)=\left(A+Q_{j}(D-A)+U_{j}(G-D)\right)-C, \\
& \bar{R}_{j}(\bar{G}-\bar{C})=\left(\bar{A}+\bar{Q}_{j}(\bar{D}-\bar{A})+\bar{U}_{j}(\bar{G}-\bar{D})\right)-\bar{C}, \\
& j=1, \ldots, N-1, \\
& \mathcal{M}_{j}: T_{j}(H-F)=\left(A+Q_{j}(D-A)+U_{j}(H-D)\right)-\left(B+S_{j}(F-B)\right), \\
& \bar{T}_{j}(\bar{H}-\bar{F})=\left(\bar{A}+\bar{Q}_{j}(\bar{D}-\bar{A})+\bar{U}_{j}(\bar{H}-\bar{D})\right)-\left(\bar{B}+\bar{S}_{j}(\bar{F}-\bar{B})\right), \\
& j=1, \ldots, N-1 .
\end{aligned}
$$

The loop equations $\mathcal{L}_{j}, \mathcal{M}_{j}$, and the normalization conditions (4) form $7(N-1)$ quadratic equations in the $2(5+3(N-1))$ unknowns consisting of the pivots locations $B, C, F, G$ and $H$ and the joint rotations $R_{j}, T_{j}$ and $U_{j}$ and their complex conjugates. The Stephenson III synthesis equations are similar in form to the Stephenson II, and both can be solved for a maximum of $N=11$ positions to obtain 70 quadratic equations in 70 unknowns that yield a total degree of $2^{70}=1.18 \times 10^{21}$.

\section{Simplification of the Synthesis Equations}

The 70 synthesis equations for the Stephenson III function generator can be reduced to 10 equations in 10 unknowns. This can be achieved by eliminating $R_{j}$ and $\bar{R}_{j}$ in the pairs of equations $\mathcal{L}_{j}$ and then eliminating $T_{j}$ and $\bar{T}_{j}$ in the pairs of equations $\mathcal{M}_{j}$ of (5). Finally, the unknowns $U_{j}$ and $\bar{U}_{j}$ can be eliminated from the resulting sets of equations, as shown in detail in Plecnik and McCarthy (2015).

To simplify the presentation of this calculation, introduce the complex numbers,

$$
\begin{aligned}
& a=G-D, \quad f=G-C, \quad h=A-C, \quad k=D-A, \\
& c=H-D, \quad g=H-F, \quad m=A-B, \quad o=-(F-B) \text {, }
\end{aligned}
$$

so the loop equations take the form

$$
\begin{array}{rlr}
\mathcal{L}_{j}: & h+Q_{j} k+U_{j} a-R_{j} f=0, & \\
& \bar{h}+\bar{Q}_{j} \bar{k}+\bar{U}_{j} \bar{a}-\bar{R}_{j} \bar{f}=0, & j=1, \ldots, 10, \\
\mathcal{M}_{j}: & m+Q_{j} k+U_{j} c+S_{j} o-T_{j} g=0, & \\
& \bar{m}+\bar{Q}_{j} \bar{k}+\bar{U}_{j} \bar{c}+\bar{S}_{j} \bar{o}-\bar{T}_{j} \bar{g}=0, & j=1, \ldots, 10 .
\end{array}
$$


Eliminate $R_{j}$ and $\bar{R}_{j}$ in $\mathcal{L}_{j}$, and $T_{j}$ and $\bar{T}_{j}$ in $\mathcal{M}_{j}$ to obtain the pairs of equations,

$$
\begin{aligned}
& \left(h+Q_{j} k+U_{j} a\right)\left(\bar{h}+\bar{Q}_{j} \bar{k}+\bar{U}_{j} \bar{a}\right)=f \bar{f} \\
& \left(m+Q_{j} k+U_{j} c+S_{j} o\right)\left(\bar{m}+\bar{Q}_{j} \bar{k}+\bar{U}_{j} \bar{c}+\bar{S}_{j} \bar{o}\right)=g \bar{g}, \quad j=1, \ldots, 10 .
\end{aligned}
$$

These 10 pairs of equations are linear in $U_{j}$ and $\bar{U}_{j}$, and can be written in the form,

$$
\left[\begin{array}{cc}
a \bar{b}_{j} & \bar{a} b_{j} \\
c \bar{d}_{j} & \bar{c} d_{j}
\end{array}\right]\left\{\begin{array}{l}
U_{j} \\
\bar{U}_{j}
\end{array}\right\}=\left\{\begin{array}{l}
f \bar{f}-a \bar{a}-b_{j} \bar{b}_{j} \\
g \bar{g}-a \bar{a}-d_{j} \bar{d}_{j}
\end{array}\right\}, \quad j=1, \ldots, 10
$$

where the complex numbers $b_{j}=D_{j}-C$ and $d_{j}=D_{j}-F_{j}$, given by

$$
\begin{aligned}
b_{j} & =h+Q_{j} k, \\
d_{j} & =m+Q_{j} k+S_{j} o, \quad j=1, \ldots, 10 .
\end{aligned}
$$

are introduced to simplify the presentation of these equations.

Eliminate $U_{j}$ and $\bar{U}_{j}$ between the pairs of equations (9) in order to obtain,

$\left.\left|\begin{array}{cc}a \bar{b}_{j} & f \bar{f}-a \bar{a}-b_{j} \bar{b}_{j} \\ c \bar{d} & g \bar{g}-c \bar{c}-d_{j} \bar{d}_{j}\end{array}\right| \begin{array}{cc}\bar{a} b_{j} & f \bar{f}-a \bar{a}-b_{j} \bar{b}_{j} \\ \bar{c} d_{j} & g \bar{g}-c \bar{c}-d_{j} \bar{d}_{j}\end{array}|+| \begin{array}{cc}a \bar{b}_{j} & \bar{a} b_{j} \\ c \bar{d} \bar{d}_{j} & \bar{c} d_{j}\end{array}\right|^{2}=0, \quad j=1, \ldots, 10$

where the vertical bars denote the determinant.

The total degree of the polynomial system in Eqn. (11) is $8^{10}=1.07 \times 10^{9}$ which is a similar case for the Stephenson II linkage Plecnik and McCarthy (2015). However, the simpler form of Eqn. (10) allows for an additional reduction before employing a multihomogeneous root count.

\section{Degree Reduction of the Synthesis Equations}

In order to reduce the degree of the synthesis equations (11), introduce the variables,

$$
\begin{array}{llll}
r_{1}=a \bar{h}, & r_{2}=c \bar{m}, & r_{3}=c \bar{o}, & r_{4}=m \bar{o}, \\
\bar{r}_{1}=\bar{a} h, & \bar{r}_{2}=\bar{c} m, & \bar{r}_{3}=\bar{c} o, & \bar{r}_{4}=\bar{m} o .
\end{array}
$$

This allows the expansion of the terms,

$$
\begin{aligned}
& a \bar{b}_{j}=r_{1}+a \bar{k} \bar{Q}_{j}, \\
& c \bar{d}_{j}=r_{2}+c \bar{k} \bar{Q}_{j}+r_{3} \bar{S}_{j}, \quad j=1, \ldots, 10 .
\end{aligned}
$$


And similarly we expand the rest of the terms in Eqn. (11) using the additional identities $f=a+h+k$ and $g=c+k+m+o$ to find,

$$
\begin{aligned}
\eta_{j}= & f \bar{f}-a \bar{a}-b_{j} \bar{b}_{j} \\
= & r_{2}+\bar{r}_{2}+r_{3}+\bar{r}_{3}+r_{4}+\bar{r}_{4}+k(\bar{g}-\bar{k})+\bar{k}(g-k) \\
& -r_{4} \bar{S}_{j}-\bar{r}_{4} S_{j}-k Q_{j}\left(\bar{m}+\bar{S}_{j} \bar{o}\right)-\bar{k} \bar{Q}_{j}\left(m+S_{j} o\right), \\
\chi_{j}= & g \bar{g}-a \bar{a}-d_{j} \bar{d}_{j} \\
= & r_{1}+\bar{r}_{1}+k(\bar{f}-\bar{k})+\bar{k}(f-k)-h \bar{k} \bar{Q}_{j}-\bar{h} k Q_{j}, \quad j=1, \ldots, 10 .
\end{aligned}
$$

See that $k$ and $\bar{k}$ are known from the specified pivot locations and $\left(Q_{j}, S_{j}\right)$, $j=1, \ldots, 10$ are known from the task requirements. Eqns. (13) and (14) are linear in terms of the unknowns $a, c, f, g, h, m, o, r_{1}, r_{2}, r_{3}$, and $r_{4}$.

The synthesis equations (11) can now be written as,

$$
\begin{aligned}
& \left|\begin{array}{cc}
r_{1}+a \bar{k} \bar{Q}_{j} & \eta_{j} \\
r_{2}+c \bar{k} \bar{Q}_{j}+r_{3} \bar{S}_{j} & \chi_{j}
\end{array}\right|\left|\begin{array}{cc}
\bar{r}_{1}+\bar{a} k Q_{j} & \eta_{j} \\
\bar{r}_{2}+\bar{c} k Q_{j}+\bar{r}_{3} S_{j} & \chi_{j}
\end{array}\right| \\
& \quad+\left|\begin{array}{cc}
r_{1}+a \bar{k} \bar{Q}_{j} & \bar{r}_{1}+\bar{a} k Q_{j} \\
r_{2}+c \bar{k} \bar{Q}_{j}+r_{3} \bar{S}_{j} & \bar{r}_{2}+\bar{c} k Q_{j}+\bar{r}_{3} S_{j}
\end{array}\right|^{2}=0, \quad j=1, \ldots, 10 .
\end{aligned}
$$

The result is a set of 10 quartic polynomials, which together with the eight quadratic polynomials, Eqn. (12), yields a polynomial system of degree, $4^{10} 2^{8}=268,435,456$.

However, the 18 unknowns in this polynomial system can be separated into the two homogeneous groups,

$$
\left\langle C, \bar{C}, G, \bar{G}, r_{1}, \bar{r}_{1}\right\rangle,\left\langle B, \bar{B}, F, \bar{F}, H, \bar{H}, r_{2}, \bar{r}_{2}, r_{3}, \bar{r}_{3}, r_{4}, \bar{r}_{4}\right\rangle \text {. }
$$

The number of roots of this system of equations can be calculated as the coefficient of $\alpha_{1}^{6} \alpha_{2}^{12}$ in the expansion of

$$
256 \alpha_{1}^{2} \alpha_{2}^{6}\left(2 \alpha_{1}+2 \alpha_{2}\right)^{10}
$$

which yields a multihomogeneous degree of 55,050,240. This is a significant reduction in the size of the polynomial homotopy needed to solve these synthesis equations. Multihomogeneous root counting is described in Bates et al. (2013). 


\section{Solution of the Synthesis Equations}

The 18 synthesis equations (12) and (15) were solved on the Gordon cluster at the San Diego Supercomputer Center of the XSEDE supercomputering network using the polynomial homotopy software BERTINI. Rather than specify the requirements for a particular task, the input parameters, $\left(Q_{j}, S_{j}\right), j=1, \ldots, 10$, were set to random complex numbers to create a numerically general system. Homotopy paths were tracked over 40 hours on $512 \times 2.6 \mathrm{GHz}$ cores. Nonsingular solutions were sorted by their Jacobian condition number of which 834,441 were found.

The roots for the general system need only be computed once, and then can be used as startpoints for a parameter homotopy for any particular set of input parameters. The advantage of parameter homotopy is that nonsingular endpoints of a general system are used as startpoints of a specific system so that only 834,441 paths need to be tracked in order to find all nonsingular solutions of a that specific system.

\section{Sorting Solutions}

The solutions of the synthesis equations are examined to determine those that represent physical linkage dimensions. This is checked by ensuring the

joint coordinate pairs $(B, \bar{B}),(C, \bar{C}),(F, \bar{F}),(G, \bar{G})$, and $(H, \bar{H})$ are complex conjugates.

Each design candidate is also evaluated to identify its cognate pair among the solutions to the synthesis equations, or to construct the cognate, if it does not appear among these solutions.

\subsection{Constructing the Cognates}

Dijksman (1976) describes how for every Stephenson III function generator, there exists one other Stephenson III function generator with link lengths of different ratios that produces the exact same function. In order to compute this function generator cognate for a Stephenson III linkage $A B C D F G H$, we consider it as a four-bar linkage $A D G C$ that controls the motion of the RR dyad $B F$ that is connected at $H$. The four-bar $A D G C$ has two other path cognates that generate the same coupler curve at $H$ (Roberts (1875)), and one of these cognates has an input link that shares the same angular displacement $\Delta \phi$ as Link $A D$ throughout the motion of $H$ (Hartenberg and Denavit (1958)). The Stephenson III function cognate is built from this four-bar path cognate. 


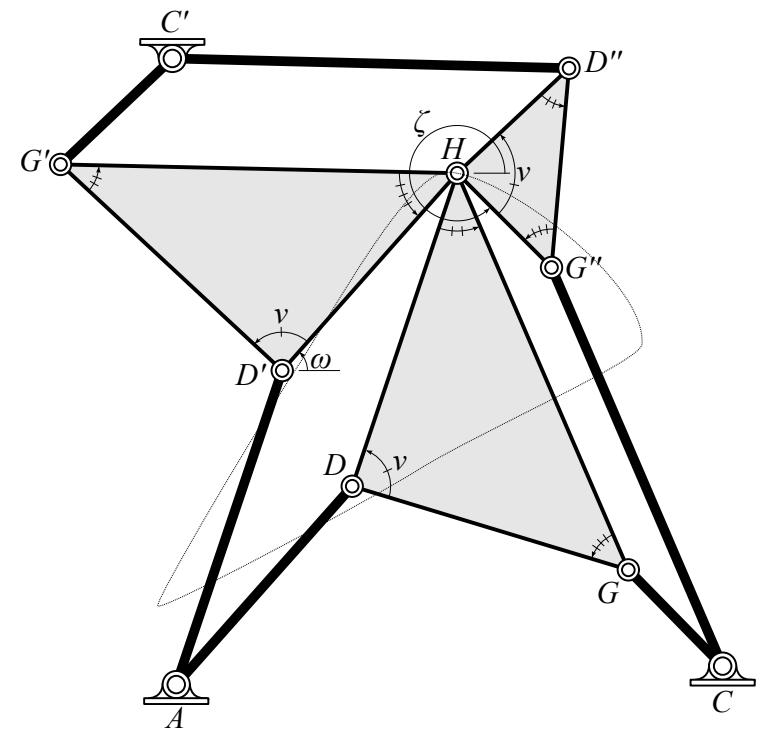

Figure 3: An overconstrained mechanism constructed from three four-bar curve cognates.

The four-bar $A D G C$ and its path cognates $A D^{\prime} G^{\prime} C^{\prime}$ and $C^{\prime} D^{\prime \prime} G^{\prime \prime} C$ are shown in Figure 3 as an overconstrained mechanism that guides the point $H$. Note that Link $C^{\prime} D^{\prime \prime}$ of cognate linkage $C^{\prime} D^{\prime \prime} G^{\prime \prime} C$ shares the same angle $\Delta \phi$ with Link $A D$ as shown in Figure 4 . Therefore, cognate linkage $C^{\prime} D^{\prime \prime} G^{\prime \prime} C$ can be connected to dyad $B F$ at $H$ to form a six-bar function cognate. Following Plecnik and McCarthy (2015), the location of pivots $C^{\prime}, D^{\prime \prime}$, and $G^{\prime \prime}$ are computed as

$$
\begin{aligned}
C^{\prime} & =\frac{(A-D)(G-H)-(C-G)(D-H)}{(G-D)}+H, \\
D^{\prime \prime} & =\left(\frac{C-G}{D-G}\right)(D-H)+H, \\
G^{\prime \prime} & =C-G+H .
\end{aligned}
$$

However, our synthesis results only contain linkages with specified pivot locations $A=0+0 i$ and $D=1+0 i$, so for the sake of comparison, we must scale, rotate, and translate the cognate linkage such that pivots $C^{\prime}$ and $D^{\prime \prime}$ line up with pivots $A$ and $D$. The transformation which computes this action on a point $p$ is

$$
\mathcal{T}(p)=\frac{D-A}{D^{\prime \prime}-C^{\prime}}\left(p-C^{\prime}\right)+A
$$




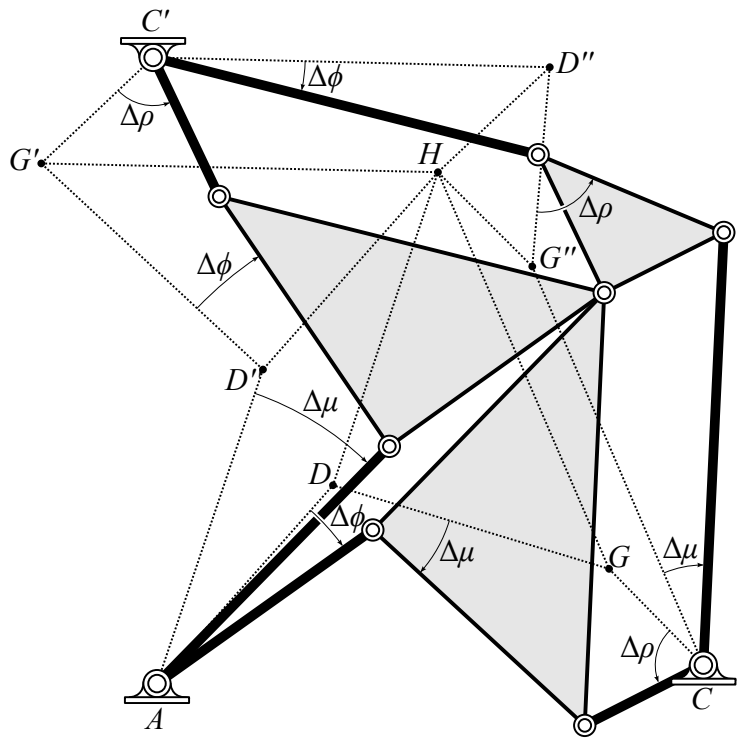

Figure 4: An overconstrained four-bar curve cognate mechanism shown in a displaced configuration.

or equivalently,

$$
\mathcal{T}(p)=\frac{D-G}{H-G}(p-C)+C
$$

Applying $\mathcal{T}$ to the cognate linkage, we find the coordinates of six-bar $(A B C D F G H)_{c}$ as

$$
\begin{aligned}
A_{c} & =\mathfrak{T}\left(C^{\prime}\right)=A \\
B_{c} & =\mathfrak{T}(B)=\frac{D-G}{H-G}(B-C)+C \\
C_{c} & =\mathfrak{T}(C)=C \\
D_{c} & =\mathfrak{T}\left(D^{\prime \prime}\right)=D \\
F_{c} & =\mathcal{T}(F)=\frac{D-G}{H-G}(F-C)+C \\
G_{c} & =\mathfrak{T}\left(G^{\prime \prime}\right)=D-G+C \\
H_{c} & =\mathfrak{T}(H)=\frac{D-G}{H-G}(H-C)+C
\end{aligned}
$$

Therefore, for every linkage solution $\{B, C, F, G, H\}$ there should exist another solution $\left\{B_{c}, C_{c}, F_{c}, G_{c}, H_{c}\right\}$ in the synthesis results. If a missing $\operatorname{cog}$ nate solution is detected, it is constructed and added to the results. 


\section{Performance Verification of a Candidate Linkage}

Once the design candidates have been sorted into cognate pairs, they are analyzed to evaluate the performance of each design. The criteria for a successful design candidate is the same as was used for the kinematic synthesis of Stephenson II function generators, Plecnik and McCarthy (2015), which is that the required accuracy points lie on a single trajectory of configurations without any singularities. Also see Chase and Mirth (1993). This is determined by computing all the configurations of the linkage for a specified range of input angles.

The kinematics equations of the Stephenson III linkage are obtained from the loop equations as,

$$
\begin{aligned}
L & =R(G-C)-(A+Q(D-A)+U(G-D))+C, \\
\bar{L} & =\bar{R}(\bar{G}-\bar{C})-(\bar{A}+\bar{Q}(\bar{D}-\bar{A})+\bar{U}(\bar{G}-\bar{D}))+\bar{C}, \\
M & =T(H-F)-(A+Q(D-A)+U(H-D))+(B+S(F-B))=0, \\
\bar{M} & =\bar{T}(\bar{H}-\bar{F})-(\bar{A}+\bar{Q}(\bar{D}-\bar{A})+\bar{U}(\bar{H}-\bar{D}))+(\bar{B}+\bar{S}(\bar{F}-\bar{B}))=0,
\end{aligned}
$$

which include the now known initial joint locations,

$$
\{A, \bar{A}, B, \bar{B}, C, \bar{C}, D, \bar{D}, F, \bar{F}, G, \bar{G}, H, \bar{H}\}
$$

and the unknown joint angle parameters,

$$
\{Q, \bar{Q}, R, \bar{R}, S, \bar{S}, T, \bar{T}, U, \bar{U}\} .
$$

In the case that the angle $\psi$ of Link $B F$ is the input parameter, then the input $\mathbf{x}$ and output $\mathbf{y}$ variables are

$$
\mathbf{x}=(S, \bar{S}), \quad \mathbf{y}=(Q, \bar{Q}, R, \bar{R}, T, \bar{T}, U, \bar{U}),
$$

and the analysis equations are

$$
\mathbf{F}(\mathbf{x}, \mathbf{y})=\left\{\begin{array}{c}
L \\
\bar{L} \\
M \\
\bar{M} \\
Q \bar{Q}-1 \\
R \bar{R}-1 \\
T \bar{T}-1 \\
U \bar{U}-1
\end{array}\right\}=\left\{\begin{array}{l}
0 \\
0 \\
0 \\
0 \\
0 \\
0 \\
0 \\
0
\end{array}\right\} .
$$


These equations have six solutions for a specified input $\mathbf{x}=(S, \bar{S})$ and are easily solved using the NSolve function in MATHEMATICA. In the case that the angle $\phi$ of Link $A D$ is the input parameter, then $\mathbf{x}=(Q, \bar{Q})$ and Eqns. (25) and (26) change appropriately. The choice of input link provides different parameterizations of the same configuration space and will define different sets of singular configurations. Singular configurations are locations in the configuration space where $\operatorname{det}\left[J_{\mathbf{F}}(\mathbf{x}, \mathbf{y})\right]=0$,

$$
\left[J_{\mathbf{F}}(\mathbf{x}, \mathbf{y})\right]=\left[\begin{array}{lll}
\frac{\partial \mathbf{F}}{\partial y_{1}} & \cdots & \frac{\partial \mathbf{F}}{\partial y_{8}}
\end{array}\right]
$$

Singular configurations define the bounds of mechanism branches.

\subsection{Sorting the linkage configurations}

A set of input parameters $\mathbf{x}_{k}, k=1, \ldots, n$ is generated that sweeps around the unit circle,

$$
\mathbf{x}_{k}=\left\{\exp \left((i 2 \pi) \frac{k-1}{n-1}\right), \exp \left(-(i 2 \pi) \frac{k-1}{n-1}\right)\right\}, \quad k=1, \ldots, n .
$$

Eqns. (26) are solved for each $\mathbf{x}_{k}$ to generate $n$ sets of configurations,

$$
\mathcal{C}_{k}=\left\{\left(\mathbf{x}_{k}, \mathbf{y}_{k, 1}\right), \ldots,\left(\mathbf{x}_{k}, \mathbf{y}_{k, 6}\right)\right\} \quad k=1, \ldots, n \text {. }
$$

The members of $\mathcal{C}_{k}$ for each $k$ appear in no particular order, and the goal of this section is to sort configurations into separate trajectories as we increment $k$ from 1 to $n$.

The algorithm initializes by setting the six elements of $\mathcal{C}_{1}$ as the beginning of six trajectories which are built upon by comparing $\mathcal{C}_{k}$ to $\mathcal{C}_{k+1}$ and deciphering pairs of connecting configurations,

$$
\begin{aligned}
& \mathcal{C}_{k}=\left\{\left(\mathbf{x}_{k}, \mathbf{y}_{k, p}\right) \mid p=1, \ldots, 6\right\}, \\
& \mathcal{C}_{k+1}=\left\{\left(\mathbf{x}_{k+1}, \mathbf{y}_{k+1, q}\right) \mid q=1, \ldots, 6\right\},
\end{aligned}
$$

where in general configurations $\left(\mathbf{x}_{k}, \mathbf{y}_{k, p}\right)$ and $\left(\mathbf{x}_{k+1}, \mathbf{y}_{k+1, q}\right)$ connect such that $p \neq q$. To decipher connections between $\mathcal{C}_{k}$ and $\mathcal{C}_{k+1}$, we use Newton's method to solve $\mathbf{F}\left(\mathbf{x}_{k+1}, \mathbf{y}\right)=0$ for $\mathbf{y}$ using start points $\mathbf{y}_{k, p}$, for $p=1, \ldots, 6$. We name these approximate solutions $\tilde{\mathbf{y}}_{k+1, p}$ where,

$$
\tilde{\mathbf{y}}_{k+1, p}=\mathbf{y}_{k, p}-\left[J_{\mathbf{F}}\left(\mathbf{x}_{k+1}, \mathbf{y}_{k, p}\right)\right]^{-1} \mathbf{F}\left(\mathbf{x}_{k+1}, \mathbf{y}_{k, p}\right), \quad p=1, \ldots, 6,
$$


is calculated from a single Newton iteration. Multiple iterations are used for more accuracy. The approximate configuration set $\tilde{\mathcal{C}}_{k+1}$ is formed from $\tilde{\mathbf{y}}_{k+1, p}$ where

$$
\tilde{\mathcal{C}}_{k+1}=\left\{\left(\mathbf{x}_{k+1}, \tilde{\mathbf{y}}_{k+1, p}\right) \mid p=1, \ldots, 6\right\} .
$$

Configuration $\left(\mathbf{x}_{k}, \mathbf{y}_{k, p}\right)$ of $\mathcal{C}_{k}$ connects to configuration $\left(\mathbf{x}_{k+1}, \mathbf{y}_{k+1, q}\right)$ of $\mathcal{C}_{k+1}$ if the following condition evaluates as true,

$$
\left|\tilde{\mathbf{y}}_{k+1, p}-\mathbf{y}_{k+1, q}\right|<\text { tol, }
$$

where tol is a specified threshold value. For most $k$, configurations $\mathcal{C}_{k}$ and $\mathcal{C}_{k+1}$ will connect in a one to one fashion. However, Eqn. (33) allows the possibility that a configuration of $\mathcal{C}_{k}$ will connect to several or none of the configurations of $\mathcal{C}_{k+1}$, which is often the case near singularities. In these cases, we employ the following logic:

1. If a configuration of $\mathcal{C}_{k+1}$ is not connected to a configuration of $\mathcal{C}_{k}$, that configuration of $\mathcal{C}_{k+1}$ begins a new trajectory.

2. If a configuration of $\mathcal{C}_{k}$ connects to multiple configurations of $\mathfrak{C}_{k+1}$, the trajectory associated with the configuration of $\mathcal{C}_{k}$ is duplicated and each duplicate connects to a matching element of $\mathcal{C}_{k+1}$.

3. If a configuration of $\mathcal{C}_{k}$ does not connect to any configurations of $\mathcal{C}_{k+1}$, the trajectory associated with the configuration of $\mathcal{C}_{k}$ is concluded.

This procedure is executed for a complete sweep of the unit circle $\mathbf{x}_{k}, k=$ $1, \ldots, n$, such that $\mathbf{x}_{n}=\mathbf{x}_{1}$. The result of this algorithm is a set of connected sequences of configurations that form separate mechanism trajectories. All combinations of these trajectories are checked for connections from $k=n$ to $k=1$ configurations. If connections are identified, these trajectories are chained together to form longer trajectories.

Finally, configurations that do not correspond to rigid body movement are removed, and the determinant of the Jacobian matrix along each trajectory is evaluated. A sign change indicates a change in trajectory that can arise from numerical error.

\subsection{Identifying successful designs}

Once all trajectories have been assembled for a linkage design candidate, each is checked to see which and how many of the specified accuracy points they contain. A successful design candidate will produce a trajectory that 
moves through all 11 accuracy points. We term these designs 11-point mechanisms.

While linkage designs that contain all 11 accuracy points on a single trajectory is the goal, our design process identifies linkage designs with trajectories that move through less than 11 points as well. It is often the case that these mechanisms only slightly miss some accuracy points and may have other features useful to the designer, such as compact dimensions or reduced link overlap.

\section{Design of a Torque Cancelling Linkage}

Survivors of strokes often suffer from a muscle control disorder called spasticity which causes increased stiffness in muscles of joints such as the wrist. Measurement data of intrinsic wrist stiffness can be found in Mirbagheri and Settle (2010), and shown in Figure 5. The goal is to design a six-bar linkage that generates a specified torque-angle profile which cancels spastic wrist stiffness. This six-bar can be incorporated into a wrist splint which passively negates the effects of spasticity.

\subsection{Obtaining the input torque profile}

The torque profile that the Stephenson III is to reproduce, and then cancel, is derived from the data collected by Mirbagheri and Settle (2010), who measured the intrinsic stiffness profile in the wrists of 21 stroke survivors. The data was taken from the graph they provided and was least-squares fit with the following fifth degree polynomial,

$$
\begin{aligned}
& S(x)=-0.3403347740344527 x^{5}+2.3767146714792213 x^{4} \\
& \quad+1.4329074166324411 x^{3}-0.21211179259258692 x^{2} \\
& \quad+0.5381754676253262 x+1.903537638831755 .
\end{aligned}
$$

Because stiffness is the rate of change of a spring torque with respect to angular deflection, we integrate $S(x)$ to obtain the torque profile,

$$
\begin{aligned}
T(x)=\int S(x) d x+c_{0} \\
=-0.056722462339075 x^{6}+0.475342934295844 x^{5}+0.358226854158110 x^{4} \\
\quad-0.070703930864196 x^{3}+0.269087733812663 x^{2} \\
\quad+1.903537638831755 x+1.859723104149862 .
\end{aligned}
$$


Eqns. (34) and (35) are graphed in Figure 5. The integration constant of the torque profile was set such that $T(-\pi / 3)=0$ which requires an unstable equilibrium at $x=-\pi / 3 \mathrm{rad}$. That means when the input link is rotated in the positive direction, a positive torque will act on it and will move the link away from the equilibrium position. Thus, this linkage will behave like a spring with negative stiffness.

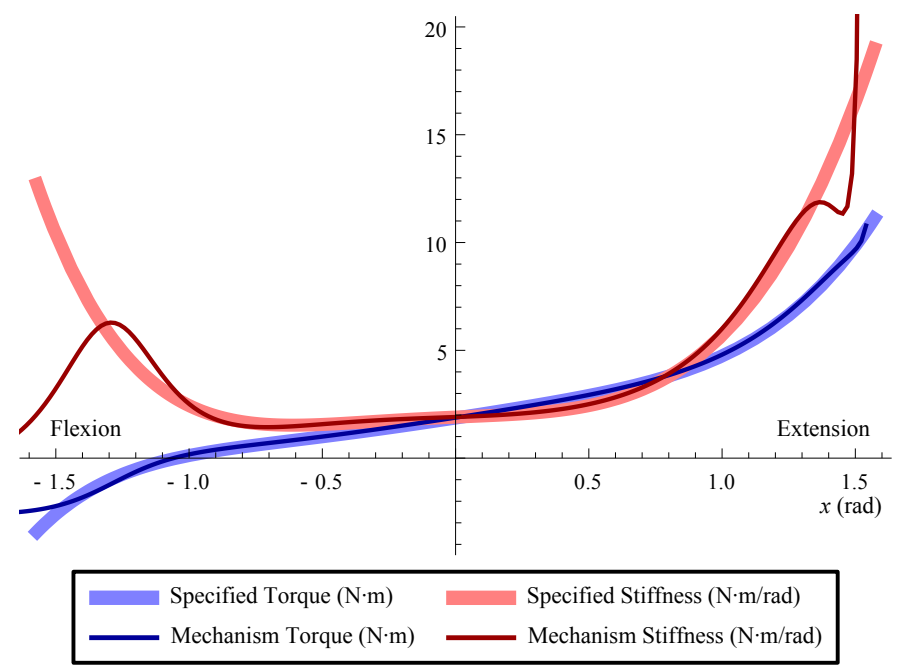

Figure 5: Desired torque and stiffness profiles as derived from Mirbagheri and Settle (2010) and the torque and stiffness profiles produced by the example mechanism. As well, the resultant torque taken by negating the mechanism generated torque from the desired torque.

\subsection{Input-output function}

The use of a function generator to provide a required input torque-angle profile begins with the assumption that there are no losses from friction, wear, and dynamic effects, which yields the power balance,

$$
T_{\text {in }} \dot{x}=T_{\text {out }} \dot{y},
$$

where $\dot{x}$ denotes the angular velocity of the input crank, and $\dot{y}$ is the angular velocity of the output crank.

For this design, the output torque $T_{\text {out }}$ is generated by a torsion spring with stiffness $k$ and equilibrium angle $y_{e}$, therefore the input torque is given by,

$$
T_{i n}=-k\left(y-y_{e}\right) \frac{\dot{y}}{\dot{x}}=-k\left(y-y_{e}\right) \frac{d y}{d x},
$$




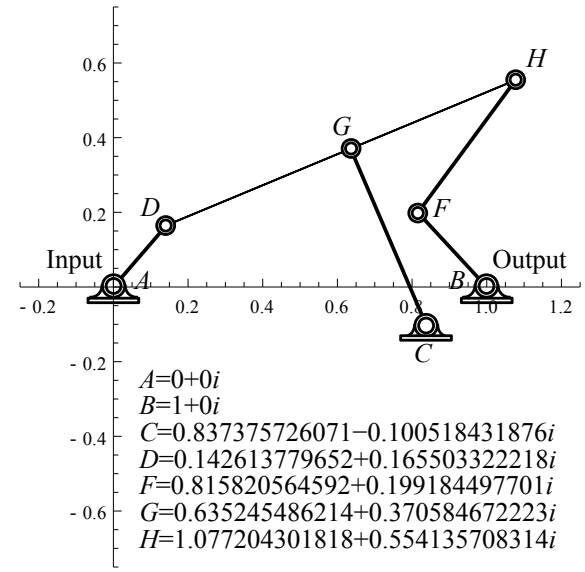

(a) $\Delta \phi$ is input

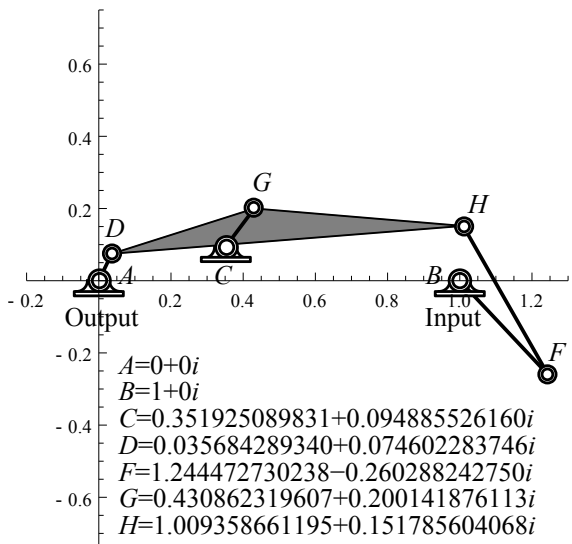

(c) $\Delta \psi$ is input

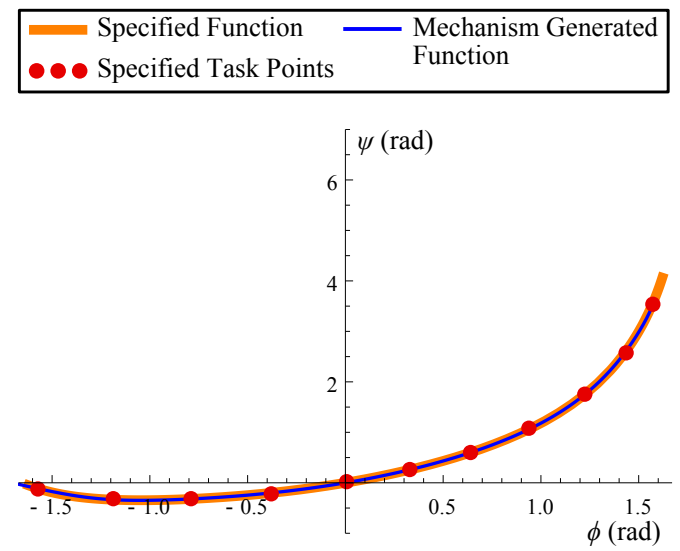

(b) $\Delta \psi=f(\Delta \phi)$

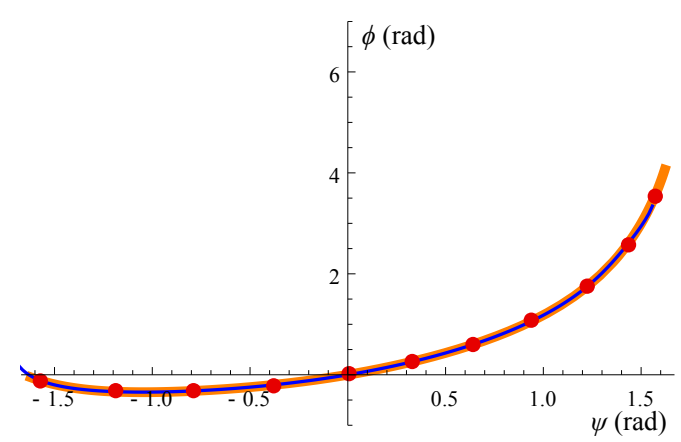

(d) $\Delta \phi=f(\Delta \psi)$

Figure 6: (a) A Stephenson III linkage actuated by $\phi$ and (b) its mechanized function and (c) a Stephenson III linkage actuated by $\psi$ and (d) its mechanized function.

which is a function of the input angle $x$. Eqn. (37) can be solved for $y=f(x)$ to obtain the set of input-output angles needed to design a Stephenson III function generator.

Separate variables and integrate to obtain,

$$
-\frac{1}{k} \int T_{i n}(x) d x=\frac{1}{2} y^{2}-y_{e} y
$$




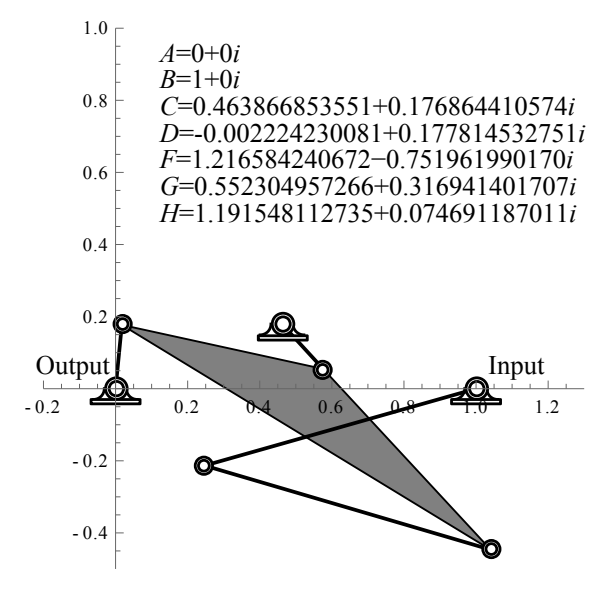

(a)

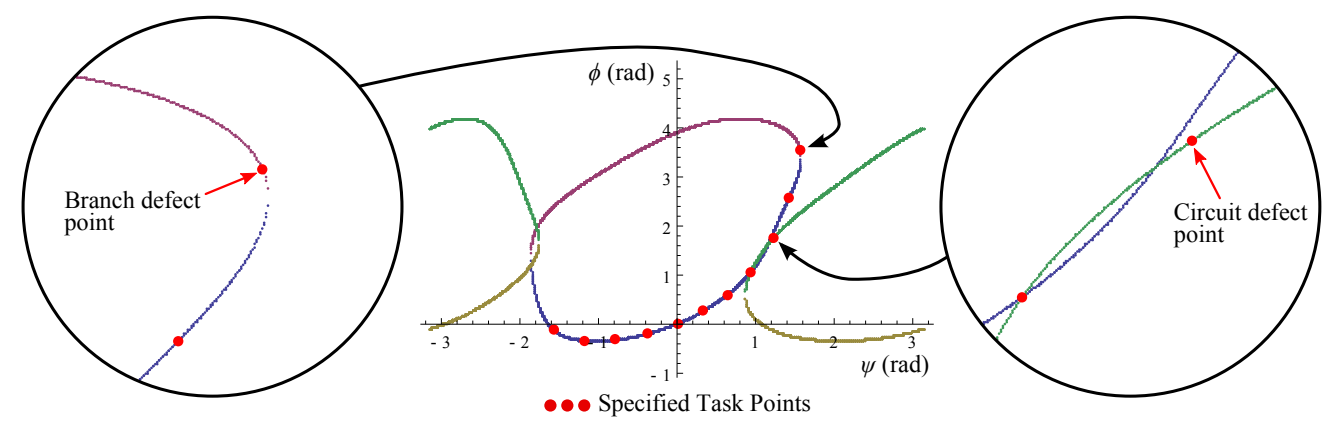

(b)

Figure 7: (a) A defective linkage and (b) its configuration space. Separate singularity free trajectories are indicated with different colors. A branch and circuit defect is illustrated.

and then solve for $y$ to obtain,

$$
y=f(x)= \pm \sqrt{-\frac{2}{k} \int T_{i n}(x) d x+y_{e}^{2}}+y_{e}
$$

The "+" and "-" solutions are two different functions that produce the desired torque profile for given spring parameters $k$ and $y_{e}$.

The input-output function for the synthesis of the Stephenson III function generator is obtained by substituting (35) into (39), with the requirement that $k=0.45 \mathrm{~N} \cdot \mathrm{m} / \mathrm{rad}$ and $y_{e}=2 \pi \mathrm{rad}$. The "-" solution was taken to calculate the input-output $y=f(x)$ function shown in Figures 6(b) and $6(\mathrm{~d})$. 
Table 1: Task position data as displayed in Figure 6.

\begin{tabular}{|r|r|r|}
\hline$j$ & \multicolumn{1}{|c|}{$x_{j}$} & \multicolumn{1}{|c|}{$y_{j}$} \\
\hline 1 & $-90^{\circ}$ & $-6.665566873543^{\circ}$ \\
\hline 2 & $-68^{\circ}$ & $-19.185437363846^{\circ}$ \\
\hline 3 & $-45^{\circ}$ & $-18.280827185669^{\circ}$ \\
\hline 4 & $-22^{\circ}$ & $-11.558672810110^{\circ}$ \\
\hline 0 & $0^{\circ}$ & $0^{\circ}$ \\
\hline 5 & $19^{\circ}$ & $15.000375068384^{\circ}$ \\
\hline 6 & $37^{\circ}$ & $34.756261256578^{\circ}$ \\
\hline 7 & $54^{\circ}$ & $61.184689516866^{\circ}$ \\
\hline 8 & $70^{\circ}$ & $99.804701760596^{\circ}$ \\
\hline 9 & $82^{\circ}$ & $148.305746675651^{\circ}$ \\
\hline 10 & $90^{\circ}$ & $203.021804302295^{\circ}$ \\
\hline
\end{tabular}

Table 2: Synthesis results for the cases of actuating Link $A D, \psi=f(\phi)$, and actuating Link $B F, \phi=f(\psi)$.

\begin{tabular}{|l|r|r|}
\cline { 2 - 3 } \multicolumn{1}{c|}{} & $\psi=f(\phi)$ & $\phi=f(\psi)$ \\
\hline Linkage solutions & 8341 & 8583 \\
\hline Cognates added & 712 & 647 \\
\hline Linkages analyzed & 4547 & 5323 \\
\hline 11 point mechanisms & 96 & 109 \\
\hline 10 point mechanisms & 225 & 131 \\
\hline 9 point mechanisms & 352 & 333 \\
\hline 8 point mechanisms & 450 & 596 \\
\hline 7 point mechanisms & 793 & 887 \\
\hline 6 point mechanisms & 1389 & 1104 \\
\hline Synthesis computation time $(\mathrm{hr})$ & 0.8 & 0.9 \\
\hline Analysis computation time $(\mathrm{hr})$ & 4.0 & 6.1 \\
\hline
\end{tabular}

This input-output function was evaluated at 11 positions of $x$ to obtain the coordinated angles shown in Table. 1. We investigate producing this function using both link $A D$ as the input, $(x, y)=(\Delta \phi, \Delta \psi)$, and link $B F$ as the input, $(x, y)=(\Delta \psi, \Delta \phi)$. The use of BERTINI to obtain solutions to the synthesis equations is the same for both cases. 


\subsection{Successful linkage designs}

A summary of synthesis results is shown in Table 2. For the cases with $\phi$ as the input and $\psi$ as the input, BERTINI found 8,341 and 8,583 solutions that corresponded to physical linkages, respectively. Each solution set was then processed to add cognate solutions and remove solutions with very small or large link lengths such that 4,547 and 5,323 solutions were found for each case. The performance of these linkages was analyzed in order to categorize mechanisms by the number of accuracy points they can achieve in a singularity-free trajectory from 6 to 11 points. For $\phi$ as the input and $\psi$ as the input, there were 96 and 109 mechanisms, respectively, that passed through all 11 points. The total computation time for each case was 5 hrs and $7 \mathrm{hrs}$ performed on $64 \times 2.2 \mathrm{GHz}$ nodes of the UC Irvine High Performance Computing Cluster.

Figure 6 shows two 11-point mechanisms and their input-output functions, one with $\phi$ as the input and one with $\psi$ as the input. Figure 5 shows the torque and stiffness profiles produced by the linkage shown in Figure $6(\mathrm{a})$.

Figure 7 illustrates some common defects found in mechanisms that achieve less than 11 accuracy points. The figure depicts the configuration of a 9 -point mechanism. Notice that one accuracy point is on a separate trajectory yielding a circuit defect. However, in the second case the accuracy point is on the same trajectory but separated from the others by a singularity, known as a branch defect. Despite these defects, the mechanism tracks the desired trajectory very closely and is useful to the designer.

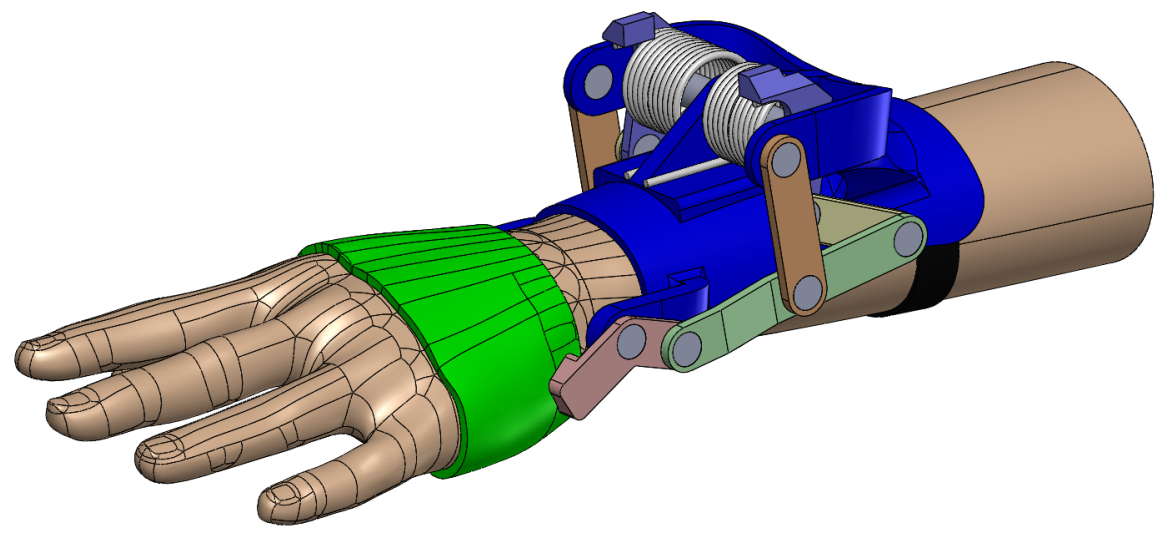

Figure 8: Solid model of the dynamic wrist splint. 


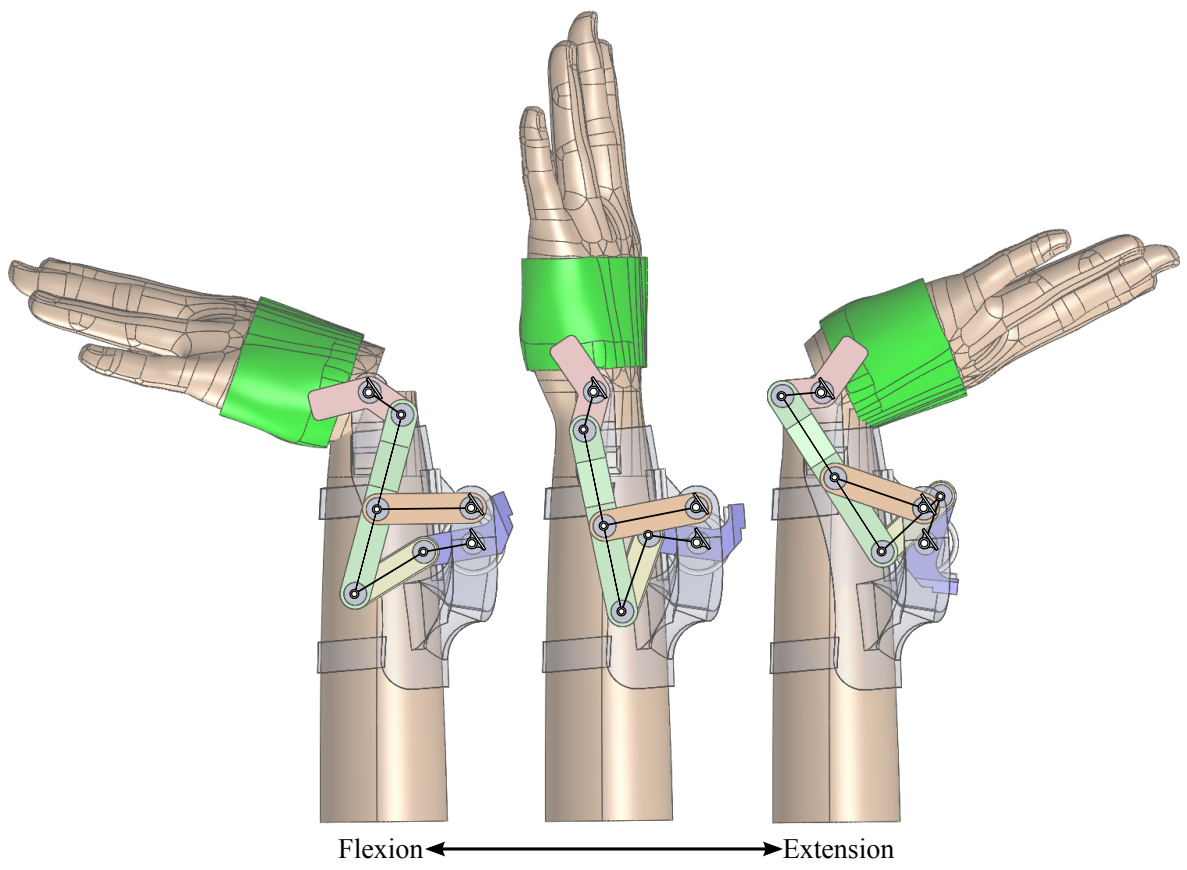

Figure 9: Solid model of the kinematic design shown in Figure 6(a) incorporated into the dynamic wrist splint.

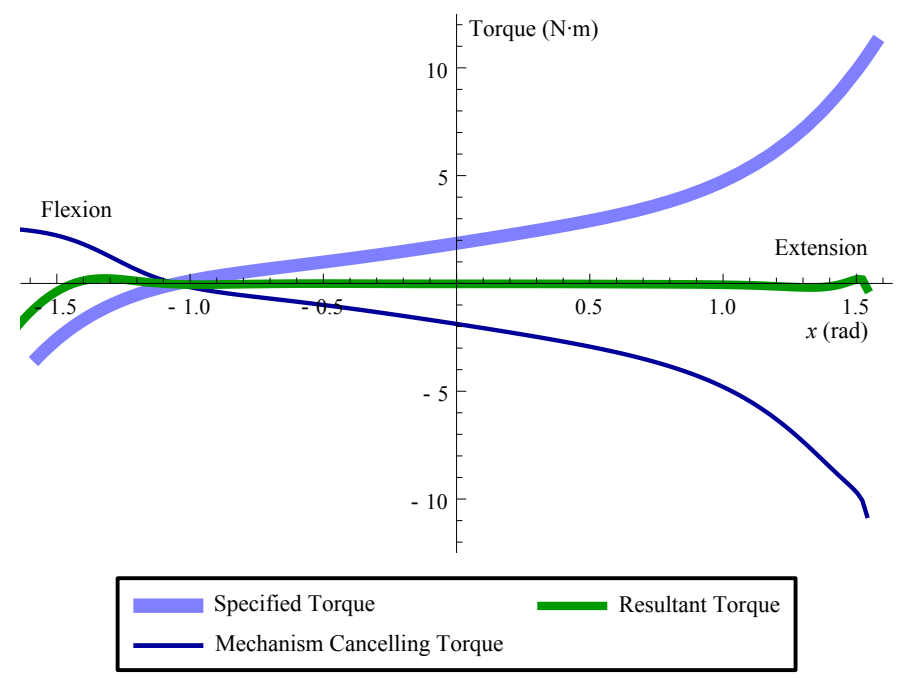

Figure 10: The resultant of the desired torque and the torque generated by the mechanism shown in Figure 6(a) is near zero. 


\subsection{Example design}

A solid model of the 11-point mechanism shown in Figure 6(a) incorporated into the design of a dynamic wrist splint is shown in Figures 8 and 9. This device can be used to cancel the torque that arises from the intrinsic stiffness of stroke survivors that suffer from spasticity, Bissell (2014). The mechanism acts against the intrinsic stiffness of the stroke survivor to reduce the torque needed for movement, as shown in Figure 10.

\section{Conclusions}

This paper presents a direct solution to the synthesis equations for a Stephenson III six-bar function generator with 11 specified input and output angles. The synthesis equations form a polynomial system with multihomogeneous degree of 55,050,240, and the polynomial homotopy software BERTINI computed 834,441 nonsingular solutions for a general set of parameters. These solutions were then used to construct a parameter homotopy to calculate the dimensions of Stephenson III six-bar function generators for a specific task. Each solution is analyzed to find the feasible designs that move through the accuracy points on a singularity-free trajectory.

This synthesis technique was demonstrated on the design of a function generator for a torque cancelling assistive wrist splint, and yielded over 16,000 linkage solutions. Of these, 205 feasible designs passed through the 11 accu-

racy points. The result is an effective design tool for Stephenson III six-bar linkages for use as function generators.

\section{Acknowledgments}

This material is based upon work supported by the National Science Foundation under Grant No. CMMI 1066082. Thank you to Jonathan Stokes for creating the solid models shown in Figs. 8 and 9.

\section{References}

I. D. Akçali, 1995. "Modular Approach to Function Generation," Proceedings of the Ninth World Congress on the Theory of Machines and Mechanisms, Pages 1440-1444, August 29-September 2, 1995, Milan, Italy.

S. S. Balli and S. Chand, 2002. "Defects in link mechanisms and solution rectification," Mechanism and Machine Theory 37(9): 851-876. 
D. J. Bates, J. D. Hauenstein, A. J. Sommese, and C. W. Wampler, 2013. Numerically Solving Polynomial Systems with Bertini, SIAM Press, Philadelphia, PA.

D. M. Bissell, 2014, WRIST (Wrist Resonator for Independent Stroke Training), M. S. Thesis, Mechanical and Aerospace Engineering, University of California, Irvine.

R. C. Blackett, 2001. Optimal Synthesis of Planar Five-link Mechanisms for the Production of Nonlinear Mechanical Advantage, M.S. Thesis, Mechanical Engineering, Virginia Polytechnic Institute and State University.

R. R. Bulatović, S. R. Đorđević and V. S. Đorđević, 2013. "Cuckoo Search algorithm: A metaheuristic approach to solving the problem of optimum synthesis of a six-bar double dwell linkage," Mechanism and Machine Theory, $61: 1-13$.

T. R. Chase and J. A. Mirth, 1993. "Circuits and branches of single-degree-offreedom planar linkages," Journal of Mechanical Design, 115(2):223-230.

A. K. Dhingra, J. C. Cheng, and D. Kohli, 1994. "Synthesis of six-link, slidercrank and four-link mechanisms for function, path and motion generation using homotopy with m-homogenization", Journal of Mechanical Design, 116(4):1122-1131.

E. A. Dijksman, 1976. Motion Geometry of Mechanisms, Cambridge University Press, London.

A. G. Erdman, G. N. Sandor, and S. Kota, 2001. Mechanism Design: Analysis and Synthesis, Prentice Hall, Upper Saddle River, NJ.

F. Freudenstein, 1954. "An analytical approach to the design of four-link mechanisms", Transactions of the ASME, 76:483-492.

R. S. Hartenberg, and J. Denavit, 1964. Kinematic Synthesis of Linkages, McGraw-Hill, New York, NY.

R. S. Hartenberg and J. Denavit, 1958. "The fecund four-bar linkage," Transactions of the Fifth Mechanism Conference, Purdue University, Lafayette, Indiana, pp. 194-206. 
W. M. Hwang and Y. J. Chen, 2010. "Defect-Free Synthesis of Stephenson-II Function Generators", Journal of Mechanisms and Robotics, 2(4):041012.

A. Liu, B. Shi, and T. Yang, 1995. "On the kinematic synthesis of planar linkages with multi-loops," Proceedings of the Ninth World Congress on the Theory of Machines and Mechanisms, Pages 95-97, August 29-September 2, 1995, Milan, Italy.

C. W. McLarnan, 1963. "Synthesis of six-link plane mechanisms by numerical analysis", Journal of Engineering for Industry, 85(1):5-10.

M. M. Mirbagheri and K. Settle, 2010. "Neuromuscular properties of different spastic human joints vary systematically", 32nd Annual International Conference of the IEEE EMBS, Buenos Aires, Argentina.

M. Plecnik and J. M. McCarthy, "Computational Design of Stephenson II Sixbar Function Generators for 11 Accuracy Points," accepted for publication in the ASME Journal of Mechanisms and Robotics, March 2015.

M. Plecnik and J. M. McCarthy, 2014. "Numerical Synthesis of Six-bar Linkages for Mechanical Computation," ASME Journal of Mechanisms and Robotics, 6(3): 031012.

S. Roberts, 1875. "Three-bar Motion in Plane Space", Proceedings of the London Mathematical Society s1-7(1):14-23.

R. R. Root and K. M. Ragsdell, 1976. "A survey of optimization methods applied to the design of mechanisms," Journal of Manufacturing Science and Engineering 98(3):1036-1041.

R. Sancibrian, 2011. "Improved GRG method for the optimal synthesis of linkages in function generation problems", Mechanism and Machine Theory, 46(10):1350-1375.

P. S. Shiakolas, D. Koladiya, and J. Kebrle, 2005. "On the optimum synthesis of six-bar linkages using differential evolution and the geometric centroid of precision positions technique," Mechanism and Machine Theory, 40(3):319-335.

P. A. Simionescu and P. Alexandru, 1995. "Synthesis of Function Generators Using the Method of Increasing the Degree of Freedom of the Mechanism," 
Proceedings of the Ninth World Congress on the Theory of Machines and Mechanisms, Pages 139-143, August 29-September 2, 1995, Milan, Italy.

P. A. Simionescu, and M. R. Smith, 2001. "Four-and six-bar function cognates and overconstrained mechanisms", Mechanism and machine theory 36(8):913-924.

L. W. Tsai, 2000. Mechanism Design: Enumeration of Kinematic Structures According to Function, CRC Press.

C. W. Wampler, 1996. "Isotropic coordinates, circularity, and Bézout numbers: planar kinematics from a new perspective," Proceedings of the 1996 Design Engineering Technical Conferences, 96-DETC/MECH-1210, Irvine, CA. 
Table(s)

\section{Table 1}

Table 1: Task position data as displayed in Figure 7.

\begin{tabular}{|r|r|r|}
\hline$j$ & \multicolumn{1}{|c|}{$x_{j}$} & \multicolumn{1}{c|}{$y_{j}$} \\
\hline 1 & $-90^{\circ}$ & $-6.665566873543^{\circ}$ \\
\hline 2 & $-68^{\circ}$ & $-19.185437363846^{\circ}$ \\
\hline 3 & $-45^{\circ}$ & $-18.280827185669^{\circ}$ \\
\hline 4 & $-22^{\circ}$ & $-11.558672810110^{\circ}$ \\
\hline 0 & $0^{\circ}$ & $0^{\circ}$ \\
\hline 5 & $19^{\circ}$ & $15.000375068384^{\circ}$ \\
\hline 6 & $37^{\circ}$ & $34.756261256578^{\circ}$ \\
\hline 7 & $54^{\circ}$ & $61.184689516866^{\circ}$ \\
\hline 8 & $70^{\circ}$ & $99.804701760596^{\circ}$ \\
\hline 9 & $82^{\circ}$ & $148.305746675651^{\circ}$ \\
\hline 10 & $90^{\circ}$ & $203.021804302295^{\circ}$ \\
\hline
\end{tabular}




\section{Table 2}

Table 2: Synthesis results for the cases of actuating Link $A D, \psi=f(\phi)$, and actuating Link $B F, \phi=f(\psi)$.

\begin{tabular}{|l|r|r|}
\cline { 2 - 3 } \multicolumn{1}{c|}{} & $\psi=f(\phi)$ & $\phi=f(\psi)$ \\
\hline Linkage solutions & 8341 & 8583 \\
\hline Cognates added & 712 & 647 \\
\hline Linkages analyzed & 4547 & 5323 \\
\hline 11 point mechanisms & 96 & 109 \\
\hline 10 point mechanisms & 225 & 131 \\
\hline 9 point mechanisms & 352 & 333 \\
\hline 8 point mechanisms & 450 & 596 \\
\hline 7 point mechanisms & 793 & 887 \\
\hline 6 point mechanisms & 1389 & 1104 \\
\hline Synthesis computation time $(\mathrm{hr})$ & 0.8 & 0.9 \\
\hline Analysis computation time $(\mathrm{hr})$ & 4.0 & 6.1 \\
\hline
\end{tabular}


Input-Output

Output

Design Candidates

Novel Devices
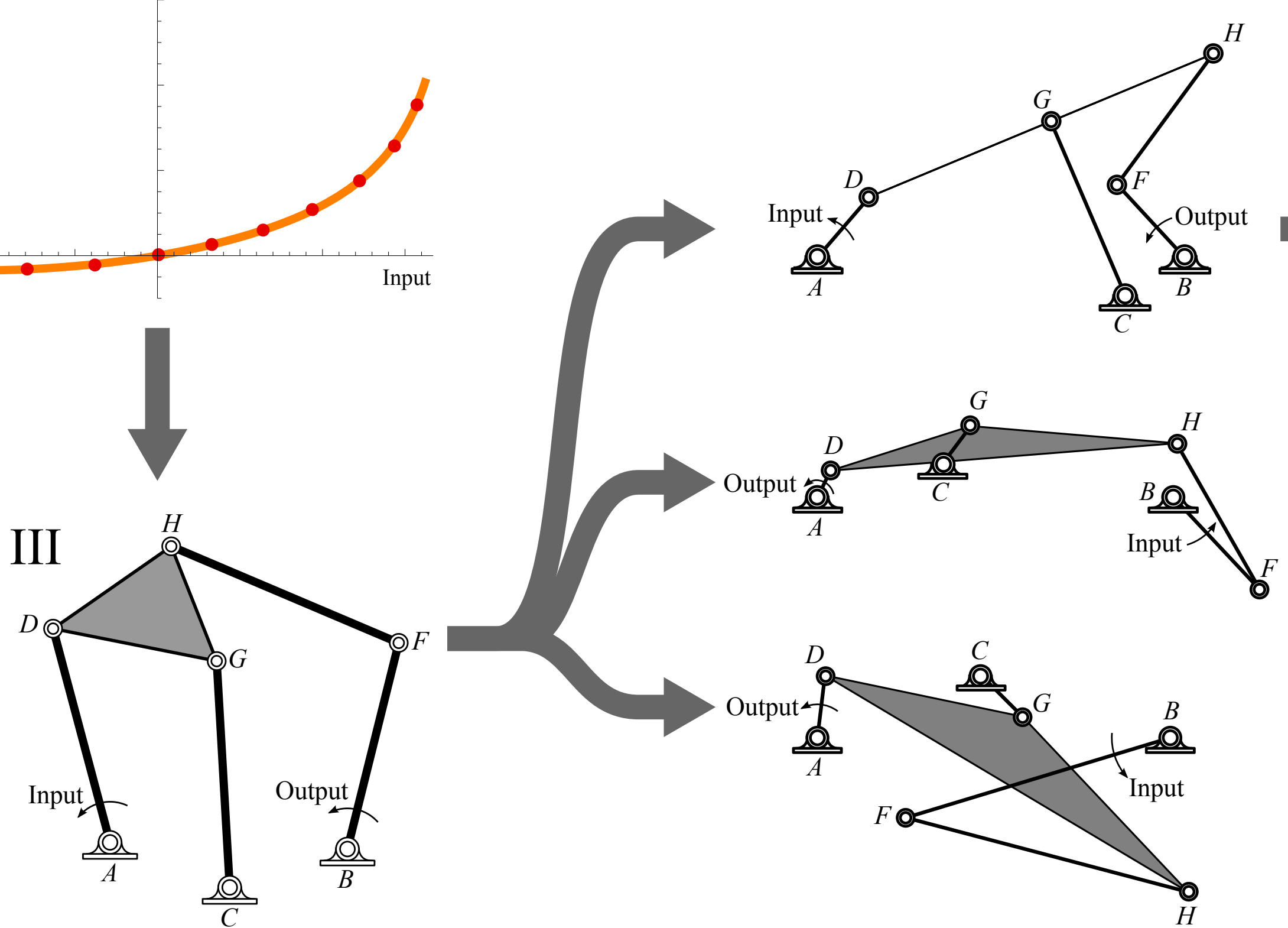

Stephenson III Synthesis
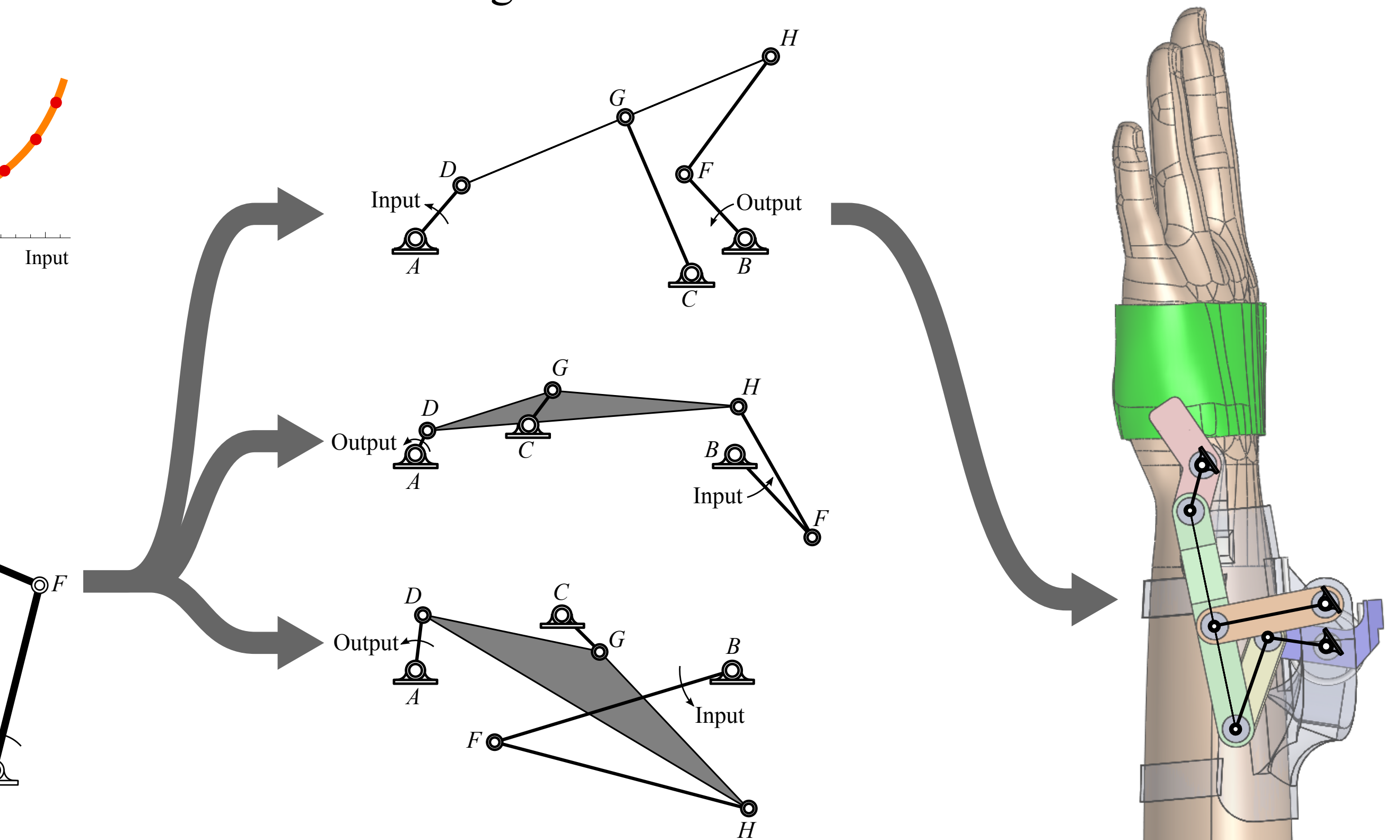